\title{
GENÓTIPO E FENÓTIPO DOS ATLETAS DE PENTATLO MILITAR MASCULINO DE ALTO RENDIMENTO NO BRASIL
}

\section{Genotype and phenotype of high level male Military Pentathlon athletes in Brazil \\ Rodrigo Ferraz Silva ${ }^{1,2}$, José Fernandes Filho ${ }^{3}$}

\section{RESUMO}

O presente estudo teve por objetivo identificar as características do perfil dermatoglífico e somatotípico, assim como das qualidades físicas básicas que caracterizam atletas de Pentatlo Militar masculino de alto rendimento, no Brasil. Ao elaborar este trabalho, considerou-se, como amostra, os seis atletas convocados pela Comissão Desportiva Militar do Brasil (CDMB), que disputaram o Campeonato Mundial de Pentatlo Militar do Conselho Internacional de Desportos Militares (CISM). A implicação prática na identificação do perfil do Pentatlo Militar, no Brasil, se coadunou com as necessidades desta modalidade esportiva, pois carecem, em nosso país, de informações sobre o esporte, tetracampeão do CISM, encontrando-se, no momento, com a sua equipe em renovação. Os dados poderão servir de parâmetros para futuras convocações e para o desenvolvimento de novos valores. O grupo foi submetido: a uma avaliação para identificação do perfil dermatoglífico, por meio das impressões digitais (ID), segundo o protocolo de Cummins e Midlo (1) ; a uma avaliação do somatotipo, pelo método antropométrico de Heath-Carter ${ }^{(2)}$; e a testes para avaliar qualidades físicas. Foram investigadas as seguintes qualidades físicas, segundo os protocolos dos testes: coordenação - Teste de Burpee; velocidade - Teste de corrida de 50 metros lançados; força explosiva de membros inferiores - Sargent Jump Test ; força explosiva de membros superiores - Two Hand Medicine Ball Put ; agilidade Shuttle Run; flexibilidade - Seat and Reach Test, Johnson e Nelson ${ }^{(3)}$; resistência aeróbica - Léger-Boucher, Léger e Boucher $^{(4)}$; e resistência anaeróbica - Teste de corrida de
40 segundos, Matsudo ${ }^{(5)}$. Na identificação do perfil dermatoglífico, foram elaboradas as características dos modelos das ID para atletas de Pentatlo Militar de alto rendimento, que incluem os índices mais informativos e integrais: $\mathrm{D} 10=9,20 \pm 2,17 ; \mathrm{SQTL}=82,3 \pm 42,34 ; \mathrm{L}=7,60 \pm$ 1,$52 ; W=0,67 \pm 0,52$; e $A=1,33 \pm 1,75$, ou seja, predominância de presilha $(L)$ e baixa presença de verticilo (W). Também ficaram estabelecidos os tipos predominantes de fórmula digital: $A L=16,7 \% ; L>W=33,3 \%$; $10 \mathrm{~L}=16,7 \%$; e ALW = 33,3\%. Na identificação do perfil somatotípico, foram apresentadas as características do grupo investigado, segundo os três componentes do somatotipo de Heath-Carter, quais sejam: endomorfia = $1,51 \pm 0,17$; mesomorfia $=5,55 \pm 1,03$; e ectomorfia $=2,2$ $\pm 0,57$. Tais valores caracterizam o grupo investigado como meso-ectomórfico. Sobre as qualidades físicas básicas, a equipe apresentou os seguintes resultados: coordenação $16,67+/-0,52$ partes; velocidade $5,81+/$ - seg; força explosiva de membros inferiores $59,33+/-6,46 \mathrm{~cm}$; força explosiva de membros superiores $5,08+/-0,21 \mathrm{~cm}$; resistência aeróbica 66,47 +/- 3,25 ml (kg.min-1); agilidade $9,03+/-0,17$ seg; flexibilidade $52+/-4,11 \mathrm{~cm}$; e resistência anaeróbica 296,50 +/- 4,04 m. Desta forma, este estudo veio apresentar o perfil da equipe brasileira de Pentatlo Militar, a fim de permitir que esta mesma população possa ser comparada, em uma outra oportunidade, consigo, com demais equipes nacionais e internacionais, e/ou sirva como um subsídio concreto para a formação esportiva do alto rendimento e a renovação do Pentatlo Militar no Brasil.

Palavras-chave: Perfil Dermatoglífico, Somatotipo, Qualidades Físicas Básicas, Pentatlo Militar.

1. Comissão Desportiva Militar do Brasil (CDMB) - Brasília/DF - Brasil.

2. Universidade Castelo Branco - Programa de Pós-graduação Stricto Sensu em Ciência da Motricidade Humana (PROCIMH) - Rio de Janeiro - RJ - Brasil.

3. Universidade Federal do Rio de Janeiro (UFRJ) - Rio de Janeiro - RJ - Brasil.

Revista de Educação Física 2008 Set; 142:28-41. Rio de Janeiro (RJ) - Brasil.

Recebido em 20.11.2007. Aceito em 14.07.2008. 


\section{ABSTRACT}

The present study aims to identify Military Pentathlon high level athletes profile about dermatoglyphic, somatotype and physical qualities. In elaborating this work, six athletes from the Brazilian military team were observed. They competed in the last edition of the world championship of the Conseil International du Sport Militaire (CISM). The practical implication, in identifying the profile of Military Pentathlon athletes in Brazil, is incorporated with the needs of this sport modality, because Brazil is four times world champion of the CISM and this team needs to get younger. Thus, Brazil needs some data to find the new athletes and to develop new values in this sport. The group was submitted to an evaluation to identify the dermatoglyphic profile by means of digital impressions (DI), following the protocol of Cummins and Midlo (1942), to an evaluation of somatotype, by the anthropometric method of Heath-Carter (1967), and tests of some physical qualities: coordination Burpee Test; speed - 50 meters launched running; leg power - Sargent Jump Test; arm power - Two Hand Medicine Ball Put, agility - Shuttle Run; flexibility - Seat and Reach Test, Johnson \& Nelson (1979); aerobic resistance Léger-Boucher, Léger \& Boucher (1980); and anaerobic resistance - 40 seconds running, Matsudo (1979). In identifying the dermatoglyphic profile, the characteristics of models of the Digital Impressions were elaborated for

\section{INTRODUÇÃO}

O Pentatlo Militar teve a sua origem em 1946, baseado em uma técnica de treinamento de unidades pára-quedistas holandesas, que, depois de lançadas de pára-quedas em uma zona demarcada, percorriam uma distância de 20 km, com obstáculos a vencer, executando, ao longo do percurso, exercícios de tiro e lançamento de granadas. Em 1947, no Centro de Treinamento Físico Militar, em Freiburg, na zona de ocupação francesa na Alemanha, realizou-se uma competição piloto e simplificada, com a eliminação do salto de pára-quedas e a reformulação das provas. O regulamento improvisado, resultante da competição, foi aprovado pelas autoridades militares francesas e as provas foram incluídas em uma competição largamente adotada por todas as forças armadas francesas, com o nome de Pentatlo Militar. O novo desporto (composto pelas provas de tiro de fuzil, pista de obstáculos, natação utilitária, lançamento de granadas e corrida de 8 high level Military Pentathlon athletes, which include the most informative and integral indexes: $D 10=9.20 \pm 2.17$; $S Q T L=82.3 \pm 42.34 ; L=7.60 \pm 1.52 ; W=0.67 \pm 0.52 ; A=$ $1.33 \pm 1.75$, with high incidence of loop $(\mathrm{L})$ and low incidence of whool (W). The predominant types of digital form were also established: $\mathrm{AL}=16.7 \% ; \mathrm{L}>\mathrm{W}=33.3 \% ; 10 \mathrm{~L}=16.7 \%$; $A L W=33.3 \%$. In identifying the somatotypic profile, the characteristics of the group studied were presented following the three components of somatotype of Heath-Carter, which were: endomorphy $=1.51 \pm 0.17$; mesomorphy $=5.55 \pm 1.03$; and ectomorphy $=2.20 \pm 0.57$. These values characterize the group studied as meso-ectomorphic. About the basic physical qualities, the results were: coordination $16.67+/-0.52$ parts; speed 5.81 +/- sec; leg power $59.33+/-6.46 \mathrm{~cm}$; arm power $5.08+/$ $0.21 \mathrm{~cm}$; aerobic resistance $66.47+/-3.25 \mathrm{ml}$ (kg.min-1); agility $9.03+/-0.17 \mathrm{sec}$; flexibility $52+/-4.11 \mathrm{~cm}$; and anaerobic resistance $296.50+/-4.04 \mathrm{~m}$. Therefore, this study came to present the profile of the Brazilian Military Pentathlon team, in order to allow the comparison of this population, at another opportunity, with its own results, or with national and international teams, and/or serve as concrete data of high level sports formation and renewal of Military Pentathlon in Brazil.

Key words: Dermatoglyphic Profile, Somatotype, Physical Qualities, Military Pentathlon.

km através campo) despertou o interesse do Conselho Internacional de Desportos Militares (CISM), que iniciou o desenvolvimento do espírito de incentivo aos diferentes países, organizando competições anuais, desde 1950. Desde então, o Campeonato Mundial de Pentatlo Militar deixou de ser realizado por apenas três vezes, em função de questões políticas ou de guerra na região onde seria realizada a competição. O Pentatlo Militar é considerado o primeiro e o mais elegante dos desportos do $\mathrm{CISM}^{(6)}$.

O Brasil participou, pela primeira vez, em 1957, obtendo um modesto $7^{\circ}$ lugar e esteve presente em quase todos os campeonatos mundiais disputados, conquistando o título de campeão nos anos de 1960, 1965, 1985, 1987, 1990, 1991 e 1994; os vice-campeonatos nos anos de 1961, 1964, 1984, 1986, 1988, 1989, 1992, 1993, 1995, 1996, 1997, 1998, 1999, 2001 e 2004, além de outros resultados expressivos. O êxito alcançado, durante as décadas de 1980 e 1990, pelos pentatletas brasileiros, garante ao Brasil 
um destaque no cenário internacional do desporto militar, dividindo com a Alemanha a hegemonia, na década de 80, e com a China, a de 90. No continente americano, o Brasil possui a total hegemonia do desporto, detendo quase todos os recordes individuais e por equipe ${ }^{(7)}$.

No campeonato mundial de Pentatlo Militar, cada país é representado por uma equipe de, no máximo, seis competidores. Determina-se a classificação geral por equipe pela soma dos pontos obtidos pelos quatro melhores atletas da equipe, ao final das cinco provas, onde cada performance obtida é transformada em pontos, por meio de tabelas específicas para cada uma das cinco modalidades ${ }^{(6)}$.

Atualmente, o Brasil encontra-se com sua equipe em renovação e não existe, no país, qualquer estudo aprofundado sobre a identificação dos perfis dermatoglífico, somatotípico e das qualidades físicas básicas que caracterizem atletas de Pentatlo Militar de alto rendimento.

O Pentatlo Militar brasileiro, apesar de apresentar excelente retrospecto e grande tradição internacional, vem envidando esforços para o aprimoramento científico das técnicas de treinamento e para o acompanhamento metodológico de seus atletas. Sua prática desportiva vem se tornando cada vez mais deficiente, haja vista a dificuldade de descobrir e de treinar novos atletas. Anualmente, é realizado apenas um campeonato nacional, que serve de seletiva para o campeonato mundial e sul-americano. Apesar disto, estudos científicos, no Brasil, sobre esta modalidade esportiva e, mais especificamente, sobre nossos atletas, são bastante incipientes. Permeando tal pensamento é que se julga primordial o conhecimento das potencialidades esportivas, pela análise das características físicas, aspectos genotípicos e qualidades físicas básicas do pentatlo, a fim de possibilitar a maximização de tais potencialidades e de descrever um modelo que sirva de base para eventuais parâmetros para o desenvolvimento do esporte.

Destarte, pesquisas com o delineamento a que esta se propôs, observando características genotípicas e fenotípicas, além de qualidades físicas, vêem-se inscritas na Ciência da Motricidade Humana, voltadas à área temática do treinamento da performance motora de alto rendimento, observando uma linha de pesquisa que aborda a dermatoglifia e somatotipia da performance motora, dentro de um projeto ainda maior, que se enquadra no eixo temático de enfoque biofísico da motricidade humana.

\section{OBJETIVO}

O objetivo do presente estudo foi identificar o perfil dermatoglífico, somatotípico, assim como as qualidades físicas básicas dos atletas brasileiros de alto rendimento na modalidade de Pentatlo Militar.

\section{REVISÃO DE LITERATURA}

O esporte de alto rendimento, no mundo, está apoiado em bases científicas que buscam aliar fatores genéticos e fenótipos, em busca de melhores performances.

As características físicas dos atletas de alto rendimento, bem como as qualidades hereditárias, as disposições psicológicas e as bases fisiológicas, estabelecidas como modelos, são de grande importância na seleção, no planejamento e no treinamento de jovens esportistas que têm talento ${ }^{(8)}$.

As impressões digitais são marcas genéticas, informativas e objetivas, que não dependem da etnia e da nacionalidade, podendo ser utilizadas, mundialmente, na prática, na seleção e na orientação desportiva precoce. Fernandes Filho ${ }^{(9)}$ acrescenta que as impressões digitais possibilitam a escolha mais adequada e a especialização no esporte, com a perspectiva de otimização quanto ao talento individual. Segundo Abramova, Nikitina e Ozolin (10), a dermatoglifia é um método simples para determinação das capacidades e possibilidades de atletas jovens. Assim, é necessário que o próprio treinador tenha domínio dos métodos mais simples de diagnóstico do potencial genético, para iniciar-se na orientação, na seleção, na descoberta de talentos, na aplicação esportiva, no treinamento específico do atleta e no desenvolvimento da resistência, da velocidade e da qualidade de força ou das ações complexas de coordenação ${ }^{(11)}$.

Nikitiuk et al. ${ }^{(12)}$ e Abramova et al. ${ }^{(13)}$ compuseram a classificação dos índices das impressões digitais com base nas análises, correlativa e fatorial, da autoclassificação multidimensional de índices da dermatoglifia, além de mais de oitenta índices somático-funcionais de atletas de alta qualificação da Rússia. Esta é constituída de cinco classes principais, que se distinguem pela dominante funcional diferente, de maneira que a intensidade baixa de desenhos (D10) e a baixa somatória da quantidade total de linhas (SQTL), se correlacionam com o alto nível de manifestações de força e de potência, mas com baixo nível de coordenação e de resistência. Ao contrário, quando 
TABELA 1

CLASSIFICAÇÃO DO CONJUNTO DOS ÍNDICES DERMATOGLÍFICOS E DOS ÍNDICES SOMÁTICO-FUNCIONAIS ENTRE ATLETAS RUSSOS DE ALTA QUALIFICAÇÃO (REMADORES, N=101).

\begin{tabular}{lcccc}
\hline Classe & \multicolumn{2}{c}{ Impressões Digitais } & \multicolumn{2}{c}{ Somático - funcionais } \\
\hline & D10 & SQTL & Mínimo & Máximo \\
\cline { 2 - 5 } & 5,5 & 26,5 & $\begin{array}{c}\text { Estatura } \\
\text { Força } \\
\text { (absoluta) } \\
\text { Resistência } \\
\text { Coordenação }\end{array}$ & Força (relativa) \\
\hline II & & Coordenação & Força \\
\hline III & 9,0 & Força (relativa) & Estatura \\
& 11,6 & Força \\
IV & & 126,4 & (absoluta) \\
\hline
\end{tabular}

Fonte: Abramova et al. ${ }^{(10)}$, adaptado pelo autor.

ocorre a elevação do nível de D10 e SQTL, há correlação com a resistência e a coordenação. Os valores máximos de D10 e de SQTL referem-se à acentuação de qualidades de coordenação dos indivíduos.

Segundo Abramova et al. ${ }^{(10)}$, a análise das impressões digitais (ID) entre atletas altamente qualificados, de diversos grupos de modalidades esportivas e de diversas posições, revelou certas tendências nas correlações dos índices integrais das ID específicas para as modalidades esportivas: modalidades esportivas caracterizadas por alta potência e tempo curto de realização demonstraram níveis baixos de D10, aumento da parcela de desenhos simples $(A, L)$ e diminuição da parcela de desenhos complicados (W, S) e do SQTL; já o alto nível de D10, a ausência de $\operatorname{arco}(A)$, o aumento da incidência de $W$ e o aumento da SQTL caracterizam modalidades esportivas de força e de coordenação e as diferenças em grupos de resistência de velocidade. Nas modalidades de jogos ocorre a mesma tendência.

Em relação à quantidade de D10 e SQTL, as modalidades de esporte de velocidade e de força relacionam-se a valores baixos de D10 e do SQTL; as de resistência, a valores intermediários; e as modalidades que possuem propriocepção complexa, a valores altos.
A ampliação do campo de atividades do jogo, ou seja, a dificuldade em realizar atividades motoras durante a prática esportiva, conjuga-se com a complexidade dos desenhos digitais e com o aumento de D10; e a quantidade de linhas, com o aumento da porcentagem de verticilos (W, S), com a redução da incidência de presilhas (L) e o desaparecimento de $\operatorname{arcos}(A)$.

Segundo Pinheiro da-Cunha ${ }^{(14)}$, aliadas às qualidades físicas, as impressões digitais são as marcas genéticas que podem servir de indicadores dos principais parâmetros de dotes e talentos motores, diferenciando, não só as características funcionais específicas para cada modalidade esportiva, mas, também, a justa especialização em uma modalidade.

A dermatoglifia tem se firmado como mais um importante vetor da complexa ciência desportiva. Quer seja como um aspecto a ser considerado no treinamento desportivo, relacionada a qualidades físicas básicas ou, em um segundo momento, na seleção de talentos, esta se tem feito presente em uma série de estudos recentes. Pesquisas envolvendo diversos esportes ${ }^{(15)}$ podem ser vistas na TABELA 2, onde estão listados os estudos recentes sobre os perfis dermatoglíficos relacionados a modalidades esportivas diversas. 
TABELA 2

PARTICULARIDADES DA DERMATOGLIFIA EM ATLETAS DE ALTO RENDIMENTO E INICIANTES.

\begin{tabular}{lccccccc}
\hline Modalidade & Ano & $\mathbf{n}$ & $\mathbf{A}$ & $\mathbf{L}$ & $\mathbf{W}$ & $\mathbf{D} 10$ & SQTL \\
\hline Soldados pára-quedistas & 2003 & 22 & 0 & $6,7 \pm 3,19$ & $3,1 \pm 3,23$ & $13,1 \pm 3,24$ & $101 \pm 19,15$ \\
Natação masculina velocistas & 2003 & 15 & $0,2 \pm 0,56$ & $7,5 \pm 1,96$ & $2,3 \pm 1,88$ & $12,1 \pm 1,96$ & $106,7 \pm 25,81$ \\
Natação feminina velocistas & 2003 & 7 & $0,9 \pm 2,27$ & $7,6 \pm 2,07$ & $1,6 \pm 1,51$ & $10,7 \pm 3,25$ & $107,7 \pm 41,17$ \\
Militares EsEFEx & 2003 & 25 & $1,8 \pm 2,27$ & $5,9 \pm 2,64$ & $2,3 \pm 2,31$ & $10,4 \pm 4,62$ & $84,0 \pm 47,72$ \\
Trampolim acrobático masculino & 2003 & 8 & $0,4 \pm 1,1$ & $6,0 \pm 2,0$ & $4,0 \pm 3,0$ & $13,0 \pm 3,0$ & $112,0 \pm 39,0$ \\
Karatê & 2003 & 14 & 0 & $4,6 \pm 1,87$ & $5,4 \pm 2,35$ & $15,4 \pm 4,08$ & $159,7 \pm 24,08$ \\
Ginástica olímpica feminina & 2002 & 25 & $0,6 \pm 1,05$ & $6,3 \pm 2,18$ & $3,1 \pm 1,45$ & $12,4 \pm 3,08$ & $97,8 \pm 25,74$ \\
Triatlo masculino & 2002 & 10 & $0,6 \pm 1,9$ & $6,3 \pm 2,99$ & $2,9 \pm 3,03$ & $12,3 \pm 4,08$ & $118,6 \pm 44,92$ \\
Pilotos de caça & 2002 & 34 & $0,3 \pm 0,0$ & $6,4 \pm 2,45$ & $3,4 \pm 2,55$ & $13,1 \pm 2,90$ & $129,4 \pm 32,10$ \\
Voleibol masculino & 2000 & 22 & $0,7 \pm 0,29$ & $6,5 \pm 2,94$ & $3,4 \pm 2,97$ & $13,4 \pm 3,11$ & $125,0 \pm 39,12$ \\
Orientação & 2003 & 8 & 0 & $5,0 \pm 2,78$ & $5,0 \pm 2,78$ & $15 \pm 2,78$ & $143,3 \pm 20,32$ \\
Futsal masculino & 2000 & 66 & $0,0 \pm 0,17$ & $6,5 \pm 2,89$ & $3,5 \pm 2,90$ & $13,5 \pm 2,93$ & $147,4 \pm 32,88$ \\
Futsal infantil & 2003 & 12 & $0,6 \pm 1,24$ & $6,5 \pm 2,15$ & $2,8 \pm 2,44$ & $12,2 \pm 3,43$ & $98,9 \pm 21,13$ \\
Futebol de campo & 2003 & 48 & $0,58 \pm 1,3$ & $6,9 \pm 2,7$ & $2,56 \pm 2,7$ & $12,0 \pm 3,2$ & $99,17 \pm 35,5$ \\
Pentatlo militar & 2003 & 6 & $1,3 \pm 1,8$ & $7,6 \pm 1,5$ & $0,7 \pm 0,5$ & $9,2 \pm 2,2$ & $82,3 \pm 42,0$ \\
Handebol feminino & 2003 & 18 & $2,6 \pm 1,51$ & $6,5 \pm 1,93$ & $1,0 \pm 1,43$ & $8,0 \pm 2,37$ & $90,0 \pm 36,31$ \\
Handebol iniciantes & 2003 & 32 & $0,2 \pm 0,74$ & $7,0 \pm 2,67$ & $2,8 \pm 2,77$ & $12,6 \pm 3,05$ & $94,6 \pm 25,08$ \\
Esgrimistas estrangeiros & 2004 & 6 & $0,2 \pm 0,41$ & $4,0 \pm 3,46$ & $5,8 \pm 3,76$ & $15,7 \pm 4,08$ & $155,8 \pm 32,44$ \\
\hline & & & & & & &
\end{tabular}

Fonte: Fernandes Filho ${ }^{(15)}$ adaptado pelo autor.

Cabe, ainda, ressaltar a importância do estudo científico e aprofundado das particularidades e das características próprias das impressões digitais dos atletas brasileiros, das diversas modalidades aqui praticadas.

Sendo assim, pode-se concluir que, no âmbito desportivo, a dermatoglifia é sustentada por vários autores que, ao longo do tempo, demonstraram o seguinte:

- os índices quantitativos e qualitativos dos desenhos das impressões digitais, com o respaldo dos autores referidos, são marcas informativas e objetivas da orientação e da seleção esportiva ${ }^{(9)}$;

- as ID sofrem alterações e mudanças nos índices dermatoglíficos, de acordo com os diferentes níveis de qualificação esportiva ${ }^{(13)}$; e

- é lícito construir um esquema de princípios da dermatoglifia, associando-a às manifestações funcionais: resistência, velocidade, coordenação, força e atividades cíclicas ${ }^{(11)}$.
A somatotipia é um instrumento utilizado para o conhecimento e identificação das características do atletas, sendo um excelente instrumento a empregar-se na descoberta de talentos, além de permitir uma contínua monitoração da composição corporal, no decorrer de uma temporada de competição ${ }^{(16)}$.

A classificação final do somatotipo é a expressão de três números que indicam o aspecto físico observado, bem como sua classificação, permitindo, ainda, a comparação direta entre dois ou mais tipos físicos.

Segundo Carter ${ }^{(17)}$, o somatotipo permite descrever e comparar desportistas em distintos níveis, caracterizar alterações do componente físico em diferentes fases da vida humana e durante o treinamento, comparar a forma relativa de homens e mulheres, servindo para a análise da imagem corporal.

O somatotipo antropométrico de Heath e Carter permite um estudo apurado sobre o tipo físico ideal para cada modalidade esportiva, sendo um excelente instrumento 
para ser empregado na descoberta de talentos, além de permitir uma contínua monitoração da composição corporal durante o decorrer de uma temporada ${ }^{(18,19)}$.

Segundo Hebbelinck ${ }^{(20)}$, os técnicos utilizam a própria experiência e intuição nos trabalhos de seleção de talentos. O método mais confiável seria a identificação do tipo de atleta ideal para cada modalidade esportiva, segundo perfis pré-determinados de atletas confirmados ${ }^{(21,22)}$.

Hebbelinck ${ }^{(20)}$ afirma que o atleta, com um físico de acordo com os padrões estabelecidos para o seu esporte, teria melhores condições de desenvolver outras qualidades (como fisiológicas e psicológicas), necessárias ao desporto de elite.

Para uma seleção esportiva criteriosa, é necessário, não apenas estabelecer um somatotipo para o desporto em questão, mas, também, determinar índices desejáveis em relação às capacidades físicas dos atletas.

Segundo Tubino e Moreira ${ }^{(23)}$, a identificação das qualidades ou valências físicas é o primeiro passo a ser feito para o desenvolvimento de uma preparação física, podendo ser, também, considerado como ponto fundamental para o êxito desejado.

A seleção de atletas, para os diferentes desportos, baseia-se na eficaz capacidade de resolver tarefas motoras de caráter técnico-tático. Esta eficiência pode ligar-se a características de resistência, de velocidade, de flexibilidade e de agilidade, aliada a fatores psíquicos estáveis e a índices somatotípicos adequados ${ }^{(9)}$.

Segundo Marins e Giannichi ${ }^{(18)}$, após determinar as qualidades físicas a serem testadas, é necessário escolher testes com coeficientes de validade, de fidedignidade e de alta objetividade, para que os resultados obtidos possam expressar um parâmetro confiável.

O Pentatlo Militar é um esporte de provas combinadas, que exige do atleta uma multiplicidade de qualidades físicas, dentre as quais se destacam: resistência aeróbica, resistência anaeróbica, força explosiva, agilidade, velocidade, flexibilidade e coordenação.

Resistência aeróbica, segundo Tubino e Moreira ${ }^{(23)}$, é a qualidade física que permite o atleta sustentar, por um período longo de tempo, uma atividade física relativamente generalizada em condições aeróbicas, isto é, nos limites do equilíbrio fisiológico, denominado steady-state.
Resistência anaeróbica é a qualidade física observada na realização de exercícios de alta intensidade e, por conseqüência, de pequena duração. Ocorre, também, de forma sistêmica ${ }^{(24)}$.

Força explosiva, ou potência, está presente em várias modalidades esportivas e requer a união de duas outras capacidades físicas: força e velocidade. Pode ser explicada pela capacidade de exercer o máximo de energia em um ato explosivo ${ }^{(18)}$.

Agilidade é uma valência física diretamente ligada à velocidade, utilizando-a como seu conceito fundamental: a velocidade de deslocamento, a velocidade de reação e a resistência de velocidade, principalmente quanto ao aspecto metabólico e à velocidade de decisão ${ }^{(25)}$.

Fauconier ${ }^{(26)}$, conforme afirmam Tubino e Moreira ${ }^{(23)}$, define velocidade como "a qualidade física particular do músculo e das coordenações neuromusculares que permite a execução de uma sucessão rápida de gestos que, em seu encadeamento, constituem uma só e mesma ação, de uma intensidade máxima e de uma duração breve ou muito breve".

Flexibilidade é a "característica de um atleta executar movimentos de grande amplitude, ou sob forças externas, ou ainda, que requeiram a movimentação de muitas articulações" (27).

Coordenação, não só é um elemento básico em uma gama muito variada de práticas esportivas, como, também, é elemento útil na vida diária doméstica e profissional, podendo ser melhorada com treinamentos, mas sempre visando uma aplicação específica.

Desta forma, Fernandes Filho ${ }^{(28)}$ declara que, no processo de avaliação física primordial ao desportista de alto rendimento, os resultados obtidos, por meio da bateria de testes utilizados, são fundamentais para que se possa desenvolver um bom programa de desenvolvimento de qualidades físicas.

\section{METODOLOGIA}

\section{População}

A população foi composta por atletas brasileiros de Pentatlo Militar, participantes do Campeonato Brasileiro de Pentatlo Militar das Forças Armadas do ano de 2004. A amostra foi composta dos seis atletas convocados pela Comissão Desportiva Militar do Brasil (CDMB) para 
FIGURA 1

PERFIL NORMALIZADO DA DERMATOGLIFIA, SOMATOTIPIA E QUALIDADES FÍSICAS BÁSICAS DOS ATLETAS DE PENTATLO MILITAR.

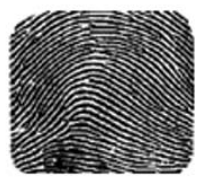

a- $\operatorname{Arco}(A)$

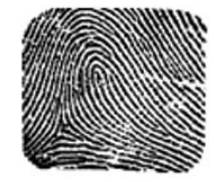

b- Presilha (L)

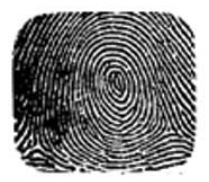

c- Verticilo (W) participarem do $52^{\circ}$ Campeonato Mundial de Pentatlo Militar de 2004 do CISM.

\section{Procedimentos}

Inicialmente, os sujeitos tomaram conhecimento dos objetivos do estudo e, após responderem a uma anamnese, assinaram o termo de consentimento, conforme as diretrizes da Resolução 196/96, do Conselho Nacional de Saúde, sobre pesquisas envolvendo seres humanos.

Em seguida, foram feitas recomendações referentes aos procedimentos regulares durante a coleta de dados. Nesta ocasião, os sujeitos foram orientados quanto aos procedimentos a serem tomados para a coleta das impressões digitais, do somatotipo e dos testes físicos relativos às qualidades físicas básicas.

\section{Dermatoglifia (protocolo de coleta das impressões digitais)}

O protocolo escolhido foi o de dermatoglifia, de Cummins e Midlo ${ }^{(1)}$, a que se refere Fernandes Filho ${ }^{\left({ }^{(9)}\right.}$. A coleta das impressões digitais foi realizada utilizando-se papel e almofada coletora de impressões da marca Finger Print Pad.

Depois da coleta das impressões digitais, deu-se o processamento preliminar dos dados, cujo método padrão é o seguinte:

- Desenhos mais comuns nas falanges distais dos dedos das mãos (FIGURA 1):

Arco "A" - desenho sem deltas - caracteriza-se pela ausência de trirrádios, ou deltas, e se compõe de cristas, que atravessam, transversalmente, a almofada digital (FIGURA 1-a);
Presilha "L" - desenho de um delta. Trata-se de um desenho meio fechado, em que as cristas da pele começam de um extremo do dedo, encurvam-se distalmente em relação ao outro, mas sem se aproximar daquele de onde se iniciam - um desenho aberto. Se a presilha está aberta para o lado radial, passa a chamar-se de "R". Se a presilha está aberta para o lado ulnar, passa a se chamar "U" (FIGURA 1-b);

Verticilo "W" - desenhos de dois deltas. Trata-se de uma figura fechada, em que as linhas centrais concentram-se em torno do núcleo do desenho (FIGURA 1-C).

- Quantidade de linhas (QL) - a quantidade de linhas das cristas de pele, dentro do desenho, é contada segundo a linha que liga o delta e o centro do desenho, sem levar em consideração a primeira e a última linha de crista.

Foram, pois, calculados os seguintes índices fundamentais das impressões digitais:

- A quantidade dos desenhos de tipos diferentes para dez dedos das mãos;

- A QL em cada dedo da mão;

- A intensidade sumária dos desenhos nos dez dedos das mãos, ou o índice de delta (D10), obtido pela soma de deltas de todos os desenhos, de modo que: a "avaliação" de Arco (A) - é sempre 0 (a ausência de delta); de cada Presilha ( $L$ ) - 1 (um delta); e de cada Verticilo (W) - 2 (dois deltas), ou seja, $\Sigma L+2 \Sigma W$; e

- O somatório total das linhas nos dez dedos das mãos.

\section{Somatotipologia}

As medidas de somatotipo foram conseguidas pelo método de Carter e Heath ${ }^{(2)}$, que permite um estudo 
apurado sobre o tipo físico ideal de cada modalidade esportiva.

Para determinação dos componentes (endomorfia, mesomorfia e ectomorfia), foram feitas as medidas das dobras cutâneas subescapular (SB), triciptal (TR) e supraespinhal (SES), utilizando-se compasso científico da marca Cescorf. Na mensuração dos diâmetros ósseos do biepicôndilo umeral (cotovelo) e do biepicôndilo femural (joelho), utilizou-se paquímetro Cescorf e, para os perímetros do braço e da panturrilha, fita antropométrica da marca Sanny. O peso corporal e a estatura foram medidos em balança e estadiômetro Filizola.

\section{Resistência aeróbica $\mathrm{VO}_{2 \text { máx }}$ (Teste de Léger-Boucher)}

O teste de Léger-Boucher ${ }^{(4)}$ foi aplicado em uma pista de atletismo oficial, balizada a cada $50 \mathrm{~m}$ com um cone (baliza). O controlador ficou no centro da pista, escutando um CD em um discman e emitindo um silvo breve de apito, a cada vez que ouve o sinal sonoro, ou dois silvos intermitentes, ao ouvir a informação de mudança de patamar. Aos avaliados, cabe somente procurar dosar sua velocidade para adequar-se aos sinais do apito.

$\mathrm{O}$ avaliado parte de qualquer uma das balizas ao ouvir o sinal de um apito, devendo estar passando a baliza seguinte no próximo sinal, prosseguindo, assim, sucessivamente, até o fim do teste. No início, os sinais são bem espaçados, de maneira a corresponderem a uma velocidade de $9 \mathrm{Km} / \mathrm{h}$. Na nomenclatura do teste é o chamado "patamar 9". A cada dois minutos de corrida, a velocidade aumenta de $1 \mathrm{Km} / \mathrm{h}$ até que o avaliado não consiga sustentar a velocidade determinada (ele começa a chegar atrasado em sucessivas balizas e não consegue recuperar o atraso), quando o teste é, então, encerrado. $\mathrm{O}$ patamar considerado como resultado do teste é o último patamar completado, acrescido, ou não, da parte fracionária cumprida pelo avaliado no patamar em que o teste foi interrompido. O resultado do teste é determinado por tabelas específicas.

\section{Resistência anaeróbica (Teste de corrida de $\mathbf{4 0}$ segundos)}

O teste em pauta tem por objetivo avaliar a resistência anaeróbica total, oriunda das vias energéticas anaeróbicas lática e alática.

Antes da execução deste teste, os avaliados foram orientados a percorrer a maior distância possível dentro dos 40 segundos de duração.
O avaliador deu início ao teste com os comandos de "Atenção! Já!", acionando, concomitantemente, o cronômetro. Um segundo avaliador esteve posicionado em um ponto médio, localizado entre 200 e 300 metros e, também, munido de um cronômetro.

O término do tempo de execução do teste foi marcado por um silvo curto de apito, sendo, naquele instante, observado o último pé do avaliado que esteve em contato com o solo, sendo marcado este ponto. O resultado foi determinado pela distância percorrida com precisão para o último metro ${ }^{(18)}$.

\section{Agilidade (Shuttle Run)}

Neste teste, cujo objetivo foi avaliar a agilidade dos atletas, foram utilizados dois blocos de madeira e um cronômetro, em um espaço livre de obstáculos de aproximadamente 15 metros, caracterizado por uma quadra poliesportiva.

O objetivo foi trazer, para trás da linha de partida, os dois blocos colocados após a linha de referência. Foram feitas duas marcas no solo, separadas, de 9,14 m, compondo as linhas de partida e de referência, depois da qual os dois blocos foram colocados. Os avaliados colocaram-se na posição semi-flexionada, com um afastamento ântero-posterior das pernas (com o pé anterior o mais próximo possível da linha de partida). Para iniciar o teste, o avaliador deu o comando "Atenção! Já!", acionando, concomitantemente, o cronômetro. Os avaliados foram orientados a correr, em sua maior velocidade possível, até o bloco, pegar um deles, retornar ao ponto de partida, depositando este bloco atrás da linha. Sem interromper a corrida, buscaram o segundo bloco, procedendo da mesma forma. $O$ teste se encerrou no momento em que o avaliado colocou o último bloco no solo e ultrapassou, com pelo menos um dos pés, as linhas que delimitavam os espaços demarcados. Os avaliados foram orientados a não jogarem os blocos, mas a colocá-los no solo. Sempre que houve erros na execução, o teste foi interrompido e repetido.

O resultado foi o tempo gasto para executar a tarefa. Foi computado o menor tempo das duas tentativas ${ }^{(3)}$.

\section{Velocidade (Teste de $\mathbf{5 0}$ metros lançados)}

Neste teste, cujo objetivo é medir a velocidade de deslocamento, foram empregados dois cronômetros para mensuração do tempo, sendo utilizada pista sintética de 
400 metros, marcada previamente em 50 metros. 0 avaliado passou sobre a linha de partida já em velocidade, iniciando o teste no momento em que se sentiu pronto. Foram necessários três avaliadores: um posicionado na linha de partida, a fim dar um sinal claro para que os outros dois avaliadores, próximos à linha de chegada, pudessem acionar o cronômetro ${ }^{(3)}$.

\section{Flexibilidade (Seat and Reach Test)}

O objetivo do teste foi medir a flexibilidade do quadril, do dorso e dos músculos posteriores dos membros inferiores.

O avaliado assumiu a posição sentada, pés apoiados no flexômetro, evitando a flexão dos joelhos. Este flexionou o quadril vagarosamente à frente, empurrando o instrumento de medida o mais à frente possível, utilizando a ponta dos dedos das mãos. O resultado computado foi o melhor de três tentativas.

\section{Força explosiva de membros superiores (Two Hand Medicine Ball Put)}

Neste teste, cujo objetivo é medir a força explosiva (potência) de membros superiores e cintura escapular, foi utilizada uma cadeira e uma bola medicinal de três quilos.

O teste foi realizado com o avaliado na posição sentada em uma cadeira, segurando uma bola medicinal de três quilos, com as duas mãos contra o peito e logo abaixo do queixo, com os cotovelos o mais próximo possível do tronco. A corda é colocada na altura do peito do avaliado a fim de mantê-lo seguro à cadeira e de eliminar a ação de embalo durante o arremesso. $O$ esforço deve ser realizado pelos braços e pela cintura escapular, evitando-se a participação de qualquer outra parte do corpo.

O resultado é computado pela distância, em centímetros, da melhor das três tentativas executadas pelo avaliado, sendo dada a ele a oportunidade de realizar uma tentativa para familiarização com o teste.

As três tentativas devem ser realizadas uma após a outra. A distância deve ser medida entre os pés dianteiros da cadeira e o primeiro ponto de contato da bola medicinal com o solo ${ }^{(3)}$.

\section{Força explosiva de membros inferiores (Sargent Jump Test)}

O objetivo deste teste é medir a potência dos membros inferiores, por meio da capacidade do impulso vertical.
Antes do início do teste, deve ser medido o peso do avaliado, além de ser aconselhável exercitá-lo por, pelo menos, cinco a 10 minutos, ministrando-lhe um aquecimento que se inicie com alongamento e termine com alguns saltos verticais.

O avaliado deve se posicionar com o seu lado dominante perto da parede, com os pés juntos. O braço oposto deve estar localizado atrás das costas, estando o outro braço voltado em direção à parede, com a mão dominante coberta de pó de giz. O avaliado terá que alcançar o mais longe que puder, esticando seus dedos e marcando o ponto mais alto, o qual deverá ser registrado.

O avaliado deve se posicionar para pular, sem movimentar os pés ou os braços durante o salto, sendo permitida a flexão dos joelhos. Uma posição de cócoras completa é adotada e, uma vez equilibrado, realizará um salto máximo. A parede é tocada com o braço totalmente estendido na altura do salto, onde o ponto mais alto é registrado.

São dadas três tentativas, sendo registrado o melhor escore da maior distância entre as duas marcas de giz ${ }^{(3)}$.

\section{Coordenação (Burpee)}

O objetivo do teste é medir a coordenação entre os movimentos de tronco, membros inferiores e superiores ${ }^{(3)}$.

O teste é dividido em quatro partes:

- partindo-se da posição de pé, flexionar os joelhos e tronco, apoiando as mãos no chão em frente aos pés;

- lançar as pernas para trás, assumindo a posição de apoio facial, braços estendidos;

- retornar, com as pernas assumindo, novamente, a posição agachada; e

- voltar à posição em pé. Ao ser dado o comando "começar", repetir a movimentação acima descrita, tão rapidamente quanto possível, até ser dado o comando "pare".

A execução do teste deve ser rigorosamente observada e sucedida de penalização de 1 ponto para as seguintes faltas:

- se os pés se moverem para trás antes que as mãos toquem o solo;

- se houver um balanço ou curvatura excessiva do quadril, quando o testando assumir a posição de apoio com os braços estendidos; 
TABELA 3

CARACTERIZAÇÃO DOS ATLETAS DE PENTATLO MILITAR.

\begin{tabular}{|c|c|c|c|c|c|}
\hline & $\mathrm{N}$ & $\mu$ & Mínimo & Máximo & $\sigma$ \\
\hline Idade (anos) & 6 & 35,33 & 31 & 43 & 4,93 \\
\hline Massa corporal (kg) & 6 & 72,28 & 62,9 & 82,8 & 8,16 \\
\hline Estatura $(\mathrm{cm})$ & 6 & 174,92 & 166 & 181 & 5,41 \\
\hline $\operatorname{Arco}(A)$ & 6 & 1,33 & 0,00 & 4,00 & 1,75 \\
\hline Presilha (L) & 6 & 7,6 & 5,00 & 10,00 & 1,52 \\
\hline Verticilo (W) & 6 & 0,67 & 0,00 & 1,00 & 0,67 \\
\hline D 10 & 6 & 9,2 & 6,00 & 11,00 & 2,17 \\
\hline Endomorfia & 6 & 1,52 & 1,32 & 1,69 & 0,13 \\
\hline Mesomorfia & 6 & 5,55 & 4,63 & 1,48 & 1,03 \\
\hline Ectomorfia & 6 & 2,20 & 6,88 & 3,08 & 0,57 \\
\hline $\mathrm{O}_{2 \max }\left[\mathrm{ml}(\mathrm{kg} \cdot \mathrm{min})^{-1}\right]$ & 6 & 66,47 & 62,5 & 70,50 & 3,25 \\
\hline Resistência anaeróbica (m) & 6 & 296,5 & 292 & 303 & 4,04 \\
\hline Agilidade (s) & 6 & 9,03 & 8,80 & 9,25 & 0,17 \\
\hline Velocidade - 50 metros lançados (s) & 6 & 5,81 & 5,51 & 6,15 & 0,24 \\
\hline Flexibilidade $(\mathrm{cm})$ & 6 & 52 & 47 & 57,5 & 4,11 \\
\hline Potência de membros superiores $(\mathrm{cm})$ & 6 & 5,08 & 4,90 & 5,40 & 0,21 \\
\hline Potência de membros inferiores $(\mathrm{cm})$ & 6 & 59,33 & 47,5 & 66,5 & 6,46 \\
\hline Coordenação (partes executadas) & 6 & 16,67 & 16 & 17 & 0,52 \\
\hline
\end{tabular}

$\sigma=$ desvio padrão, $\mu=$ média.

- se retirar as mãos do chão antes que assuma, novamente, a posição agachada (posição número 3); e

- se a posição em pé não for ereta, cabeça para cima ${ }^{(18)}$.

\section{RESULTADOS E DISCUSSÃO}

Foram analisados os desenhos das ID, seus tipos de combinação, o D10, a QL, bem como a SQTL. A somatotipologia foi fracionada em seus três componentes de endomorfia, mesomorfia e ectomorfia. Observou-se, também, os valores alcançados nos testes relativos às qualidades físicas eleitas como básicas do esporte.

As TABELAS 3 e 4 contêm os resultados médios, mínimos e máximos, assim como os desvios da caracterização da amostra, dos desenhos das impressões digitais, D10, QL, SQTL, da somatotipologia e das qualidades físicas básicas.

A amplitude da idade de 13 anos e a elevada média de idade de 35,33 anos demonstram a influência do fator experiência na obtenção de excelentes resultados, bem como a interação existente entre os atletas mais jovens e aqueles mais experientes.

Sobre as qualidades físicas básicas, o valor médio do $\mathrm{VO}_{2 \text { máx }}$ alcançado demonstra muito boa resistência aeróbica, concretizada com a segunda e terceira colocações na prova de corrida através campo, nos campeonatos mundiais de 2003 e 2004, respectivamente. Em relação ao resultado da resistência anaeróbica, os atletas de Pentatlo Militar $(296,5 \pm 4,04 \mathrm{~m})$ apresentam resultados semelhantes aos atletas de Atletismo (295,90 $\pm 17,70 \mathrm{~m})^{(5)}$. Sobre a agilidade, os resultados apresentaram valores próximos ao Futsal ${ }^{(29)}$. Sobre a potência de membros superiores, os valores encontrados enquadram-se no nível de performance intermediário, segundo Marins e Giannichi ${ }^{(18)}$. No que diz respeito à potência de membros inferiores, a média apresentada foi de $59,33 \pm 6,46 \mathrm{~cm}$, muito semelhante ao Futsal, com $59,50 \pm 4,55 \mathrm{~cm}$. Não foram encontrados, na literatura pesquisada, resultados de testes das demais qualidades físicas avaliadas, para uma amostra semelhante, a fim de 
TABELA 4

DERMATOGLIFIA DOS ATLETAS DE PENTATLO MILITAR.

MESQL1 MESQL2 MESQL3 MESQL4 MESQL5 MDSQL1 MDSQL2 MDSQL3 MDSQL4 MDSQL5 SQTL

\begin{tabular}{lccccccccccc}
$\mathrm{N}$ & 6 & 6 & 6 & 6 & 6 & 6 & 6 & 6 & 6 & 6 & 6 \\
$\mu$ & 11,8 & 6,5 & 5,7 & 8,5 & 7,2 & 12,8 & 5,8 & 8,0 & 8,8 & 7,2 & 82,3 \\
$\sigma$ & 6,79 & 5,47 & 5,32 & 2,35 & 4,07 & 8,13 & 5,98 & 4,20 & 4,71 & 3,82 & 42,34 \\
$\mathrm{SE}$ & 2,77 & 2,23 & 2,17 & 0,96 & 1,66 & 3,30 & 2,44 & 1,71 & 1,92 & 1,56 & 17,28 \\
MíNIMO & 0 & 0 & 0 & 7 & 2 & 0 & 0 & 3 & 0 & 1 & 20,00 \\
MÁXIMO & 21 & 16 & 13 & 13 & 12 & 23 & 15 & 14 & 14 & 11 & 143,0 \\
\hline
\end{tabular}

$\sigma=$ desvio padrão, $\mu=$ média e $\mathrm{SE}_{\mathrm{M}}=$ erro padrão.

que se efetuasse o pertinente escalonamento. No entanto, os valores, ora destacados, são fundamentais para a realização de comparações futuras.

Pela análise dos valores referentes à dermatoglifia, percebe-se que os atletas de Pentatlo Militar têm predominância pelo desenho digital L, moderados índices de D10 e do SQTL, enquadrando-se entre os níveis II e III da classificação de índices dermatoglíficos e somato-funcionais, proposta por Abramova et al. ${ }^{(30)}$. As qualidades físicas de força, de velocidade, de potência e de estabilidade estática, sintomáticas em desportos de alta potência e curto tempo de duração, são percebidas pelo tipo de desenho digital e pela quantidade de linhas. No entanto, características coordenativas e de força relativa poderiam mostrar-se menos desenvolvidas, caso não tenham sido estimuladas nas adequadas fases do desenvolvimento motor, por meio da realização de treinamentos específicos. No GRÁFICO 1, observa-se os valores normalizados das variáveis estudadas.

No GRÁFICO 2, tem-se os valores relativos ao somatotipo dos atletas. Observa-se que os atletas apresentam, como média, 1,52 \pm 0,13, 5,55 \pm 1,03, 2,2 \pm 0,57 , caracterizando-os como meso-ectomórfico. Em relação a outros esportes, por não existir resultados sobre o Pentatlo Militar, os resultados obtidos assemelham-se com os dos atletas de provas de velocidade no Atletismo, 100 e 200 metros rasos, e com os do Decatlo ${ }^{(2)}$, que possui as mesmas características do Pentatlo Militar, já que é um esporte de provas combinadas.

\section{GRÁFICO 1}

\section{PERFIL NORMALIZADO DA DERMATOGLIFIA, SOMATOTIPIA E QUALIDADES FÍSICAS BÁSICAS DOS ATLETAS DE PENTATLO MILITAR.}

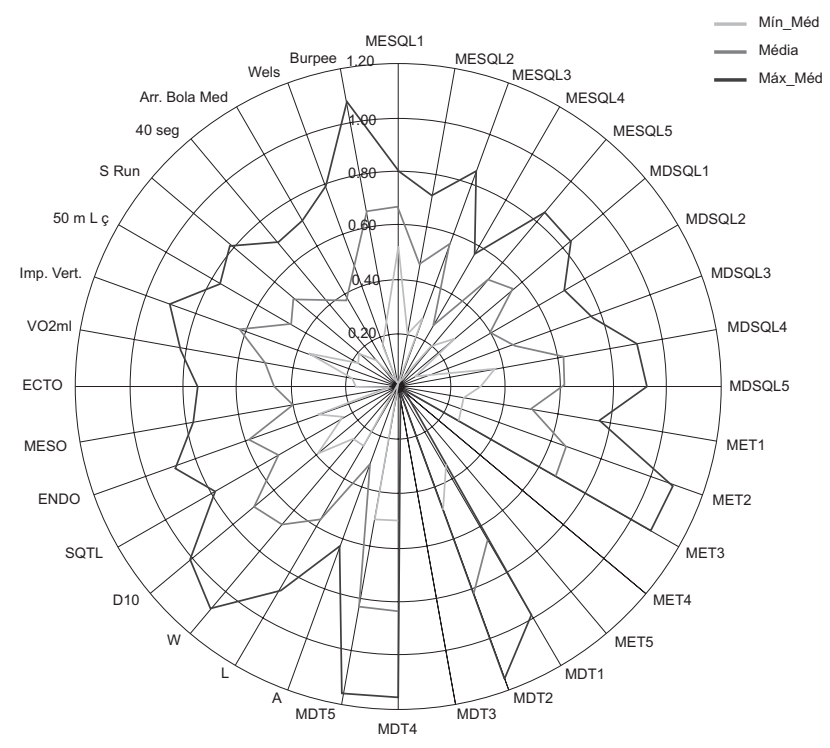




\section{GRÁFICO 2 \\ SOMATOCARTA DOS ATLETAS DE PENTATLO MILITAR.}



\section{CONCLUSÃO}

A pesquisa realizada buscou, por meio dos índices qualitativos e quantitativos, representados pelas ID, pelas características antropométricas evidenciadas por meio da somatotipologia e pelos valores das qualidades físicas básicas, descrever o perfil da equipe brasileira de Pentatlo Militar, levantando características de marcas informativas, objetivas e de orientação da seleção esportiva.

De uma maneira geral, não há, no Brasil, e provavelmente no mundo, nenhum estudo científico sobre este tema, sendo este o primeiro estudo da equipe tetracampeã mundial de Pentatlo Militar, que, apesar da idade avançada de alguns atletas, ainda possui elevado nível de competitividade. Destarte, uma comparação mais aprofundada dos resultados obtidos, na presente pesquisa, com outras equipes do mundo, tornou-se inviável.
Os valores encontrados, nos testes aplicados para mensurar as qualidades físicas básicas, podem não traduzir a performance apresentada em uma competição. Tal afirmativa justifica-se na impossibilidade de mensurar a qualidade física desejada e o gesto motor aplicado nas provas, o nível de concentração, o estado emocional dos atletas, dentre outros fatores durante as competições. No entanto, os resultados obtidos pela equipe estudada foram considerados pertinentes aos testes aplicados, sagrando-se a equipe vice-campeã mundial de 2004.

Sendo assim, espera-se que os dados obtidos referentes a dermatoglifia, a somatotipia e as qualidades físicas de resistência aeróbica, de potência de membros inferiores, de velocidade, de agilidade, de resistência anaeróbica lática, de potência de membros superiores, de flexibilidade e de coordenação, assim como outras informações referentes ao esporte em questão, possam servir de parâmetro descritivo e comparativo para estudos relacionados à preparação física, técnica e tática, à seleção e detecção de talentos, à procedimentos de iniciação esportiva, entre outros.

Desta forma, este estudo veio apresentar o perfil da equipe brasileira de Pentatlo Militar, a fim de permitir que esta mesma população possa ser comparada, em uma outra oportunidade, consigo, com demais equipes nacionais e internacionais, e/ou sirva como um subsídio concreto da formação esportiva do alto rendimento e renovação do Pentatlo Militar no Brasil.

Espera-se que esta pesquisa venha a motivar novos estudos sobre o Pentatlo Militar, incentivando sua prática, tornando-o ainda mais popular e, além disso, podendo o Brasil voltar a ocupar o primeiro lugar no ranking do CISM.

\section{REFERÊNCIAS BIBLIOGRÁFICAS}

1. Cummins H, Midlo C. Palmar and plantar dermatoglyphics in primates. Philadelphia: Winstar Institute of Anatomy and Biology; 1942.

2. Carter JEL, Heath BH. Somatotyping: development and applications. New York: Cambridge University Press; 1990.

3. Johnson BL, Nelson JK. Practical measurements for evaluation in physical education. 3rd ed. Edina: Burgess Publishing; 1979.

4. Léger L, Boucher R. An indirect continuous running multistage field test: the Université de Montréal Track Test. Can J Appl Sport Sci 1980; 5:77-84.

5. Matsudo VKR. Testes em ciências do esporte. 4ª ed. São Caetano do Sul: Gráficos Burti; 1987.

6. Conselho Internacional do Desporto Militar (CISM). Regras gerais do Pentatlo Militar - Parte A; 2003. 
7. Escola de Educação Física do Exército (EsEFEx). A geração de ouro e prata do Pentatlo Militar brasileiro. Revista de Educação Física 1990; 118: 28-38.

8. Wang W. Neurotypes and identification of sport talent. Sports-science 1989; 9(3):71-5.

9. Fernandes Filho J. Impressões dermatoglíficas - marcas genéticas na seleção dos tipos de esporte e lutas (a exemplo de desportista do Brasil) [dissertação]. Moscou, URSS: Instituto de Investigação Científica de Cultura Física e Esportes da Rússia; 1997.

10. Abramova TF, Nikitina TM, Ozolin NN. Possibilidades das impressões dermatoglíficas no prognóstico dos potenciais energéticos nos atletas que praticam remo acadêmico / Atualidades na preparação de atletas nos esportes cíclicos. In: Coletânea de artigos científicos. Volvograd 1995: 57-61.

11. Abramova TF, Nikitina TM, Ozolin NN. Impressões dermatoglíficas nos atletas de alta qualificação de diferentes esportes. Problemas morfológicos nos esportes. Volvograd 1992: 8-14.

12. Nikitiuk BA. Pesquisas da medicina esportiva. Novidades e técnicas científicas. Série Antropologia. Moscou 1990: 24; 51.

13. Abramova TF, Nikitina TM, Ozolin NN. Impressões dermatoglíficas: marcas genéticas no potencial energético do homem. In: Anais científicos do ano. Moscou; 1996: 3-13.

14. Pinheiro da-Cunha RS, Fernandes Filho J. Identificação do perfil dermatoglífico de atletas estrangeiros de alto rendimento das três armas, participantes do Campeonato Mundial de Esgrima, Havana - Cuba/2003. Fitness \& Performance Journal 2004;3(5): 247-53.

15. Fernandes Filho J. A prática da avaliação física. $2^{\underline{a}}$ ed. Rio de Janeiro: Shape; 2003.

16. International Society for the Advancement of Kinanthropometry - ISAK. Apostila de curso. Rio de Janeiro: ISAK; 2000.

17. Carter JEL. Somatotipia. In: Norton K, Olds T. Antropometrica. $3^{\underline{a}}$ ed. Rosario: Biosystem Servicio Educativo; 2000 : 134-55.

18. Marins JCB, Giannichi R. Avaliação e prescrição de atividade física: guia prático. 2ª ed.Rio de Janeiro: Shape; 1998.

19. Pavel DAC, Fernandes Filho J. Identificação dos perfis dermatoglífico, somatotípico e das qualidades físicas básicas de atletas de alto rendimento na modalidade de natação em provas de meio-fundo e fundo. Fitness \& Performance Journal 2004; 3(1):18-27.

20. Hebbelinck M. Identificação e desenvolvimento de talentos no esporte: relatos cineantropométricos. Revista Brasileira de Ciência e Movimento 1989; 4(1).

21. Silva RF, Zary JCF, Pinheiro da-Cunha RS, Martins MEA, Ferreira AAM, Lincoln AT, et al. Perfil dermatoglífico e somatotípico da equipe brasileira de Pentatlo Militar participante do 51을 Campeonato Mundial de Pentatlo Militar do CISM. In: XXVI Simpósio Internacional de Ciências do Esporte. São Paulo: CITTA Gráfica; 2003: 222.

22. Teixeira A, et al. Estudo descritivo sobre a importância da avaliação funcional como procedimento prévio no controle fisiológico do treinamento físico de futebolistas realizado em pré-temporada. Acta Fisiátrica 1999; 6(2):70-7.

23. Tubino MJG, Moreira SB. Metodologia científica do treinamento desportivo. 13ª ed. Rio de Janeiro: Shape; 2003.

24. Dantas EHM. A prática da preparação física. 5ª ed. Rio de Janeiro: Shape; 2003.

25. Manso JMG, Valdivielso MN, Caballero JAR. Bases teóricas del entrenamiento deportivo. Madrid: Gymnos; 1996.

26. Fauconnier L. L'Éntraínement Moderne. Bruxelles: Administration de L'Éducation Physique, des Sports et de la Vie en Pleine Air, Ministère de L'Éducation Nationale et de la Culture; 1978. 
27. Weineck J. Treinamento ideal. 9ª ed. São Paulo: Manole; 1999.

28. Fernandes Filho J. Descoberta de talentos [CD-ROM]. Rio de Janeiro, RJ: Ed. Shape; 2003.

29. Dantas PMS, Fernandes Filho J. Identificação dos perfis genéticos, de aptidão física e somatotípico que caracterizam atletas masculinos, de alto rendimento, participantes do Futsal adulto, no Brasil. Fitness \& Performance Journal 2002; 1(1): 28-36.

30. Abramova TF, Nikitina TM, Chafranova El. Impressões dermatoglíficas - marcas genéticas na seleção nos tipos de esportes. Atualidades na preparação de atletas nos esportes cíclicos. In: Coletânea de artigos científicos. Volvograd 1995: 86-91.

\section{Endereço para correspondência:}

Rodrigo Ferraz Silva

SMU QRO Casa 501

Brasília/DF - Brasil

CEP 70.630-000

e-mail: msrodrigoferraz@gmail.com

\section{PUBLIQUe seUS ARTIGOS NA REVISTA dE EDUCAÇÃO FísICA}

- Primeira Revista de Educação Física do Brasil.

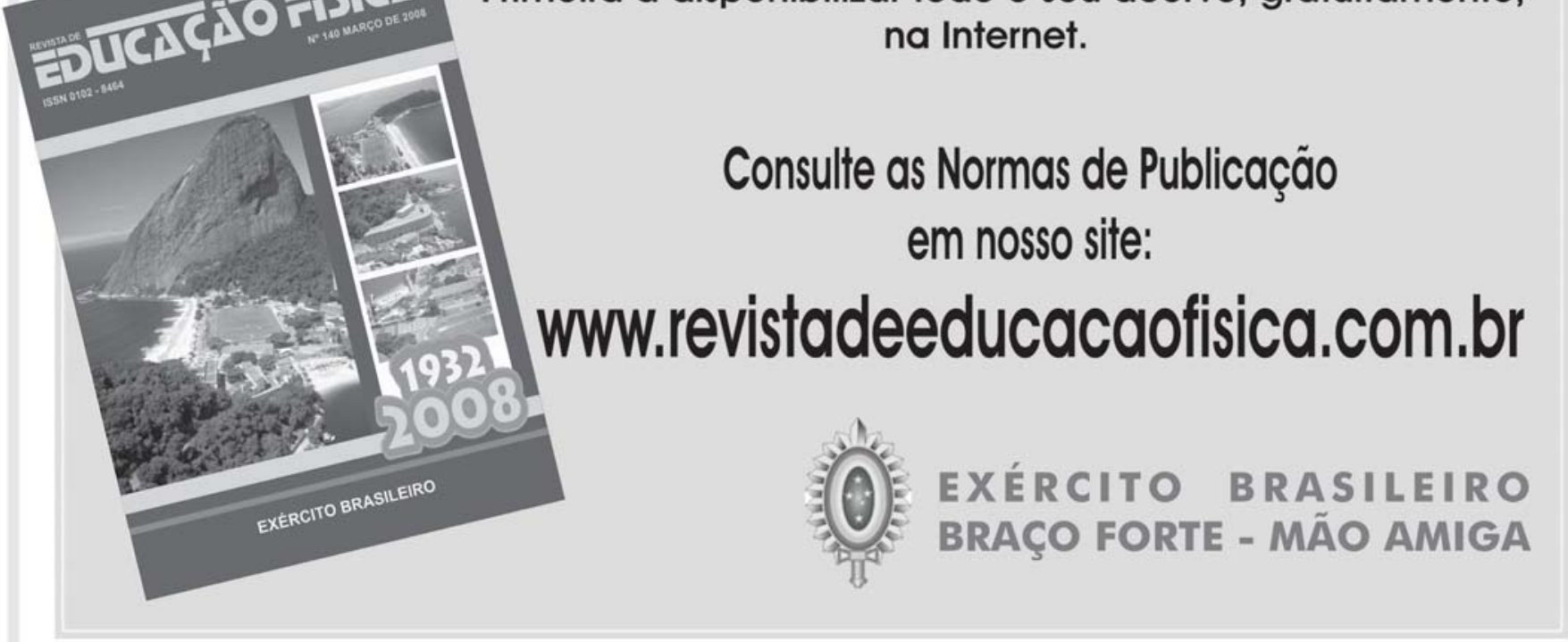

